\title{
Exosome EpCAM promotes the metastasis of glioma by targeting the CD44 signaling molecule on the surface of glioma cells
}

\author{
Quan Gu${ }^{A-C, F}$, Xuelin Chen ${ }^{B, C, F}$, Lin Zhou ${ }^{B, C, F}$, Xianglu Liu ${ }^{A, D-F}$ \\ Department of Neurosurgery, Fudan University Affiliated Zhongshan Hospital Qingpu Branch, Shanghai, China \\ A - research concept and design; B - collection and/or assembly of data; $C$ - data analysis and interpretation; \\ $\mathrm{D}$ - writing the article; $\mathrm{E}$ - critical revision of the article; $\mathrm{F}$ - final approval of the article
}

Address for correspondence

Xianglu Liu

E-mail: |x|191218@yeah.net

Funding sources

None declared

Conflict of interest

None declared

Received on May 9, 2020

Reviewed on May 14, 2020

Accepted on August 5, 2020

\begin{abstract}
Background. Glioma, the most common primary tumor in the central nervous system, originates from glial cells and has a poor prognosis.

Objectives. This experimental laboratory study was designed to explore the role of epithelial cell adhesion molecule (EpCAM) in the metastasis of glioma.

Material and methods. Serum samples were collected from patients with non-metastatic or metastatic glioma ( $n=20$ per group), and healthy volunteers $(n=8)$. Exosomes were isolated from the serum and the morphological characteristics were observed under a scanning electron microscope (SEM). The expression of CD81 and CD63 was measured to identify exosomes. Glioma tissue and the adjacent normal tissue samples were obtained from patients with non-metastatic or metastatic glioma ( $n=12$ per group). Meanwhile, 4 normal brain tissue samples were collected. The expression of CD44, hyaluronan-mediated motility receptor (HMMR), and matrix metalloproteinase-9 (MMP-9) was determined in each group using immunohistochemistry. The protein expression of CD44, HMMR, matrix metalloproteinase-2 (MMP-2), MMP-9, and selectin E (SELE) was measured with western blotting.

Results. Exosomes were present in the serum, and the proteins CD81 and CD63 were expressed in all 3 groups. CD44 was highly expressed in the non-metastasis and metastasis groups. The expression of HMMR and MMP-9 in the Adj-metastasis and Adj-non-metastasis groups was high, while in the other groups, the levels were low. The expression of CD44 in the metastasis and non-metastasis groups was significantly higher than that of the negative control (NC) group, and the expression in the metastasis group was higher than that of the non-metastasis group. The MMP-2 and MMP-9 were not found in either the metastasis or non-metastasis group. The protein expression of HMMR and SELE was high in all groups.
\end{abstract}

Conclusions. Exosome EpCAM promoted the metastasis of glioma by targeting CD44.

Key words: metastasis, CD44, glioma, exosome, epithelial cell adhesion molecule

Cite as

Gu Q, Chen X, Zhou L, Liu X. Exosome EpCAM promotes the metastasis of glioma by targeting the CD44 signaling molecule on the surface of glioma cells. Adv Clin Exp Med. 2020;29(11):1277-1282. doi:10.17219/acem/126051

DOI

10.17219/acem/126051

\section{Copyright}

Copyright by Author(s)

This is an article distributed under the terms of the

Creative Commons Attribution 3.0 Unported (CC BY 3.0)

(https://creativecommons.org/licenses/by/3.0/) 


\section{Introduction}

Glioma is the most common primary tumor in the central nervous system; it originates from glial cells and has a poor prognosis. ${ }^{1}$ Glioma is classified into 4 grades according to the latest classification of the World Health Organization (WHO). ${ }^{2,3}$ Although glioma can be treated with advanced chemotherapy drugs, surgery, new generations of gamma rays, and other interventions, the invasiveness of glioma and the protective effect of the bloodbrain barrier remain obstacles in glioma treatment. Normal brain tissue is more prone to radiation brain damage, resulting in a five-year mortality rate for glioma patients that remains at over $95 \% .{ }^{4}$ In recent years, some researchers have come to believe that the occurrence of tumors is closely related to the interaction between external environmental factors and the host's internal genetic factors. The discovery of a proto-oncogene and tumor suppressor gene have led to a more profound understanding of the mechanism of tumor occurrence and development. ${ }^{5}$ The research on the occurrence and development mechanism of brain glioma has been raised to the molecular level. Studies have found that many cytokines and genes participate in the occurrence and development of brain glioma, and that these cytokines and genes have broad application prospects in clinical practice.

Epithelial cell adhesion molecule (EpCAM) is a single transmembrane protein which is encoded by the tumorassociated calcium signal transduction gene 1 (TACSTD1) and which belongs to the family of adhesion molecules. ${ }^{6}$ It participates in the regulation of intercellular adhesion, signal transduction, and cell migration, proliferation and differentiation. ${ }^{7}$ The EpCam is expressed in the epithelium of different organs and tumors in different sites. ${ }^{8}$ In carcinomatous cells, EpCAM is overexpressed. ${ }^{9} \mathrm{Cimi-}$ no et al. found that EpCAM was upregulated in breast cancer metastasis. ${ }^{10}$ It was also found that the expression of EpCAM in metastatic gastric cancer (GC) was higher than in primary GC. The downregulation of EpCAM by siRNA led to the inhibition of GC cell invasion and migration. ${ }^{11}$ The EpCAM expression is related to tumor prognosis and may be used for tumor-targeted therapy. Stoecklein et al. showed that the mean recurrence interval for patients with strongly EpCAM-positive esophageal squamous cell carcinoma was 9 months, while the mean recurrence interval of EpCAM-negative, weakly EpCAM-positive or -positive patients was 43 months, indicating the poor prognosis of patients with overexpressed EpCAM. ${ }^{12}$

In this study, we explored the role of EpCAM in glioma and its relationship with CD44 on the surface of glioma cells.

\section{Material and methods}

\section{Tissue and serum samples}

In this experimental laboratory study, 20 patients with non-metastatic glioma and 20 patients with metastatic glioma admitted to Chifeng College Affiliated Hospital, China, from May 2016 to April 2018 were enrolled. Eight healthy volunteers were also recruited as the negative control group (NC). This study was approved by the ethics committee of Chifeng College Affiliated Hospital and all patients signed informed consent forms. Fasting peripheral venous blood ( $3 \mathrm{~mL}$ ) was drawn from all participants, placed into EDTA anticoagulant test tubes (Sigma-Aldrich, St. Louis, USA), and centrifuged at $1000 \times \mathrm{g}$ for $10 \mathrm{~min}$. The supernatant was transferred into an RNA-enzyme-free centrifuge tube (Sigma-Aldrich) and stored at $-20^{\circ} \mathrm{C}$ for later use.

Samples of glioma tissue and adjacent normal tissue were collected from 12 non-metastatic patients and 12 metastatic patients. Meanwhile, normal brain tissue samples were collected from 4 healthy subjects. All tissues were frozen immediately after resection and stored at $-80^{\circ} \mathrm{C}$ for further experiments.

\section{Exosome extraction and identification}

Exosomes were isolated from human serum using an exosome separation reagent (Invitrogen, Carlsbad, USA). In order to remove impurities, $500 \mu \mathrm{L}$ of serum was centrifuged at $2000 \times \mathrm{g}$ for $30 \mathrm{~min}$. Then, $300-\mu \mathrm{L}$ samples were mixed with $60 \mu \mathrm{L}$ of separation reagent and incubated at $4^{\circ} \mathrm{C}$ for $30 \mathrm{~min}$. After centrifugation at $10,000 \times \mathrm{g}$ for $10 \mathrm{~min}$, the supernatant was discarded. Next, $150 \mu \mathrm{L}$ of phosphate-buffered saline (PBS) was added to the suspended sediment and placed in storage at $4^{\circ} \mathrm{C}$ for later use. The exosomes were fixed with paraformaldehyde (Sigma-Aldrich), added to the copper mesh, and negatively stained with uranium dioxide-acetate (Invitrogen). The morphology was observed using a transmission electron microscope (Nikon, Tokyo, Japan).

\section{Immunohistochemistry}

The paraffin-embedded sections were routinely dewaxed to water, soaked in 3\% hydrogen peroxide (Sigma-Aldrich) at room temperature for $5 \mathrm{~min}$ to inactivate endogenous enzymes, and washed thrice in distilled water for $3 \mathrm{~min}$ each time. The antigen was thermally remediated, cooled and washed twice with PBS. The sections were then treated with $5 \%$ bovine serum albumin (BSA) sealant and placed at room temperature for $20 \mathrm{~min}$, incubated with primary antibody (rabbit IgG, 1:50 dilution; Abcam, Cambridge, UK) at $37^{\circ} \mathrm{C}$ for $60 \mathrm{~min}$, and washed 3 times with PBS. Then, the sections were incubated with secondary antibody (Abcam) at $37^{\circ} \mathrm{C}$ for $1 \mathrm{~h}$, colored with 3,3'-diaminobenzidine (Sigma-Aldrich), rinsed, and counter-stained with hematoxylin. 


\section{Western blotting}

The tissue samples were lysed with radioimmunoprecipitation assay (RIPA) lysate (Bio-Rad, Hercules, USA) on ice, and the supernatant was collected after centrifugation at $12,000 \times \mathrm{g}$ for $20 \mathrm{~min}$ at $4^{\circ} \mathrm{C}$. The protein concentration was determined with a BCA kit (Shanghai Biyuntian, Shanghai, China). SDS-PAGE electrophoresis (Merck Millipore, Burlington, USA) was performed to isolate the protein after mixing it with the buffer solution. Then, it was transferred to polyvinylidene fluoride (PVDF) membrane, sealed with a sealant, incubated overnight with CD44, matrix metalloproteinase-2 (MMP2), MMP9, selectin E (SELE), and ERBB2 primary antibodies (Abcam) at $4^{\circ} \mathrm{C}$, and incubated with horseradish peroxidase (HRP)labeled secondary antibody (Abcam) at room temperature for $2 \mathrm{~h}$. After treating the samples with chemiluminescent substrate working fluid (Merck Millipore), the membrane was developed in a Tanon 5200 chemiluminescence analysis system (Thermo Fisher Scientific, Waltham, USA).

\section{Statistical analysis}

The statistical analysis was done in SPSS v. 19.0 software (IBM Corp., Armonk, USA). The t-test was used to identify any differences between the 2 groups. A p-level $<0.05$ was considered statistically significant.

\section{Results}

\section{Morphology of exosomes}

The exosomes were extracted from the blood of the patients and the healthy volunteers. The findings of transmission electron microscopy (Fig. 1) revealed that the exosomes in the non-metastasis and metastasis groups were round or nearly round, with a double-layer lipid molecular structure, uneven size and distribution, and a diameter of 20-200 $\mathrm{nm}$. These findings confirmed that exosomes existed in the serum of the glioma patients.

\section{Exosome identification}

Western blotting was applied to determine the protein expression of CD81 and CD63, the specific surface markers of exosomes. As shown in Fig. 2, the proteins CD81 and CD63 were expressed in all 3 groups, which further confirmed that exosomes were abundant in the serum of patients with glioma.

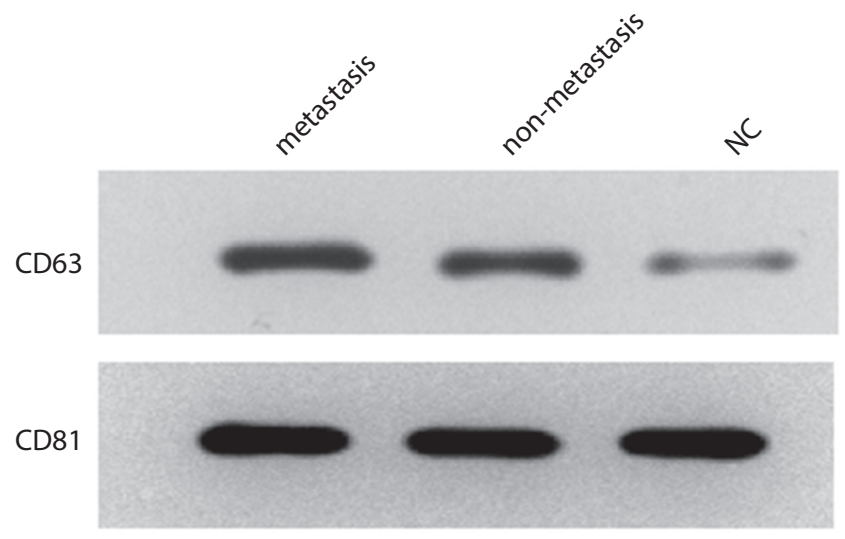

Fig. 2. The protein expression of 2 specific surface markers, exosomes CD81 and CD63, was measured with western blotting

\section{CD44, HMMR, and MMP9 expression in non-metastatic and metastatic gliomas}

Immunohistochemistry was performed to measure the expression of CD44, hyaluronan-mediated motility receptor (HMMR) and MMP-9 in the metastasis, adjacent(Adj)metastasis, non-metastasis, Adj-non-metastasis, and NC groups. As shown in Fig. 3, CD44 was highly expressed in the non-metastasis and metastasis groups, though it was expressed very little in the other groups. The expression of HMMR and MMP-9 in the Adj-metastasis group was the highest, followed by the Adj-nonmetastasis group. Other groups demonstrated a low expression of HMMR and MMP-9.
NC

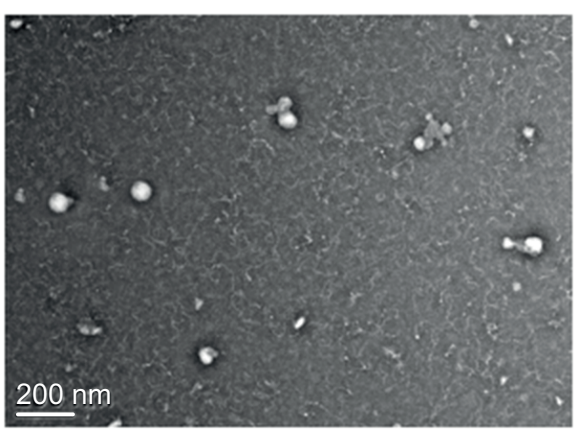

non-metastasis

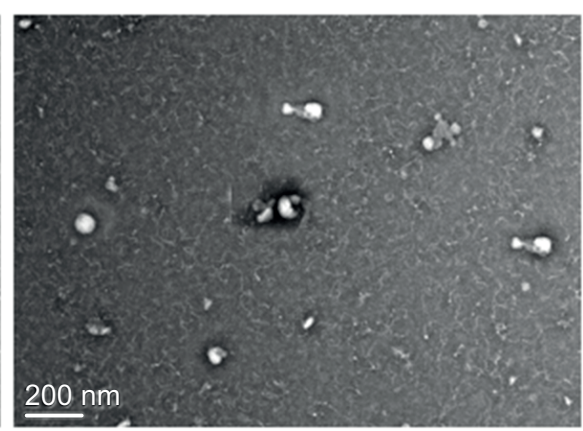

metastasis

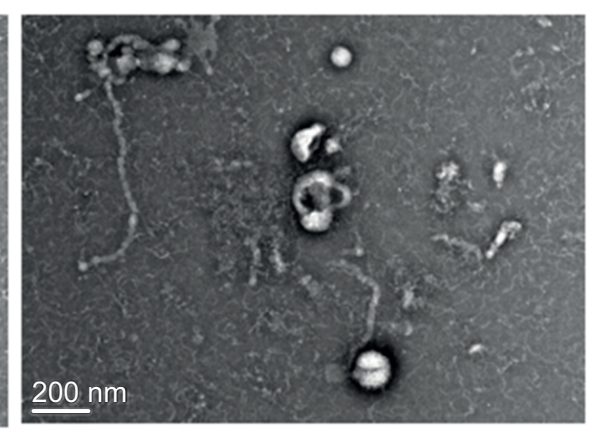

Fig. 1. The morphology of exosomes was examined using a transmission electron microscope 


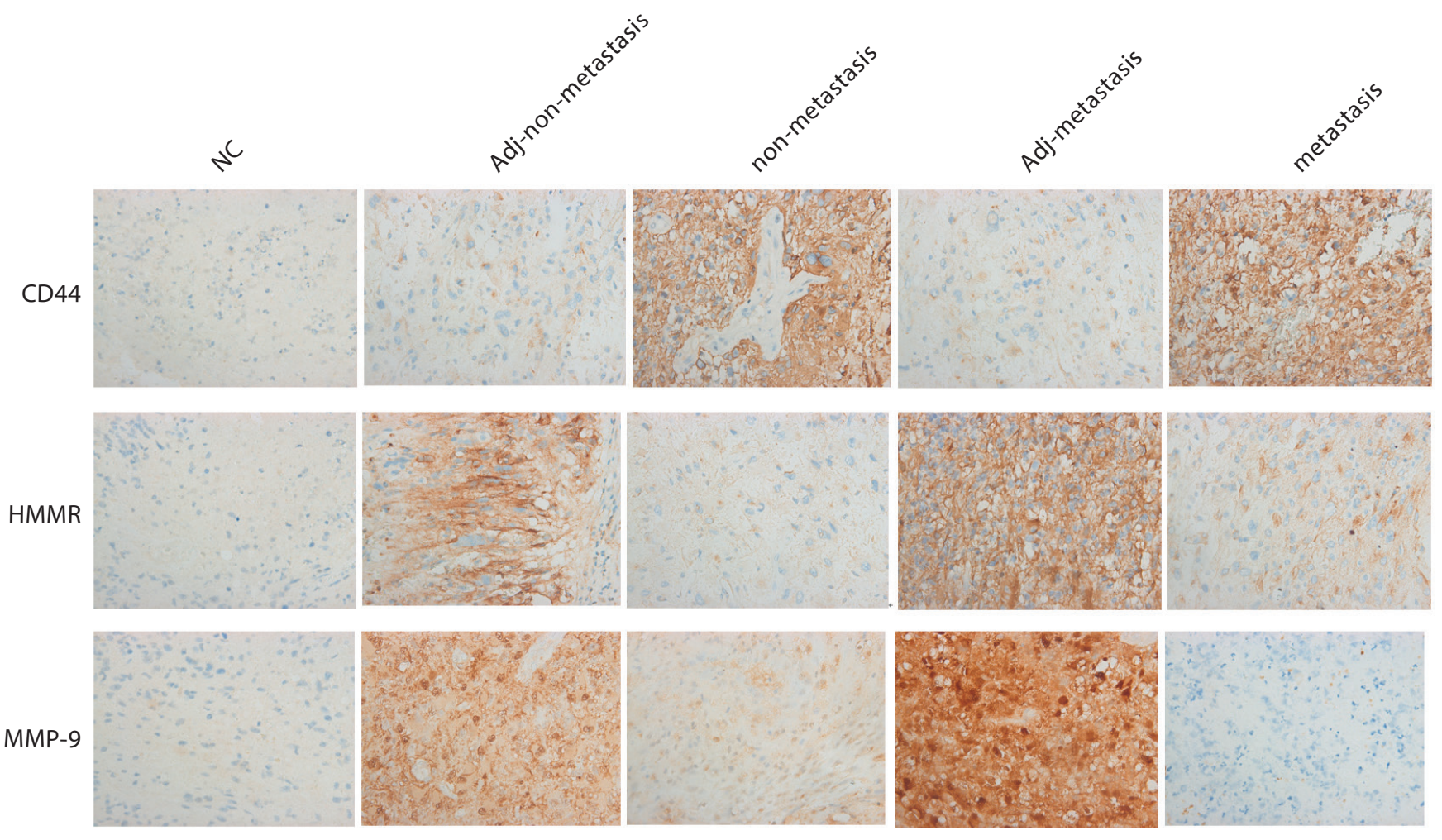

Fig. 3. The expression of CD44, HMMR and MMP-9 in the metastasis, Adj-metastasis, non-metastasis, Adj-non-metastasis, and NC groups was determined using immunohistochemistry assay

\section{Metastasis influences gene expression}

The protein expression of CD44, HMMR, MMP-2, MMP-9, and SELE in the metastasis, Adj-metastasis, nonmetastasis, Adj-non-metastasis, and NC groups were measured. As shown in Fig. 4, CD44 expression in the metastasis and non-metastasis groups was significantly higher than in the NC group, while it was not expressed in the Adjmetastasis or Adj-non-metastasis groups. Moreover, the expression of $\mathrm{CD} 44$ in the metastasis group was higher than in the non-metastasis group. The MMP-2 and MMP-9 were not expressed in the metastasis or non-metastasis groups, and they were expressed to a much higher degree in the Adjmetastasis and Adj-non-metastasis groups than in the NC group. The expression of HMMR and SELE in the metastasis, Adj-metastasis, non-metastasis, and Adj-non-metastasis groups were all higher than the expression in the NC group. In addition, HMMR and SELE showed a much higher expression in the Adj-metastasis and Adj-non-metastasis groups in comparison to the metastasis and non-metastasis groups.

\section{Discussion}

Glioma is often invasive and the boundaries between the tumor and surrounding tissue are blurred, making it difficult to remove completely during surgery. ${ }^{13}$ At present, the clinical treatment of glioma remains unsatisfactory, so recent studies have focused on the development

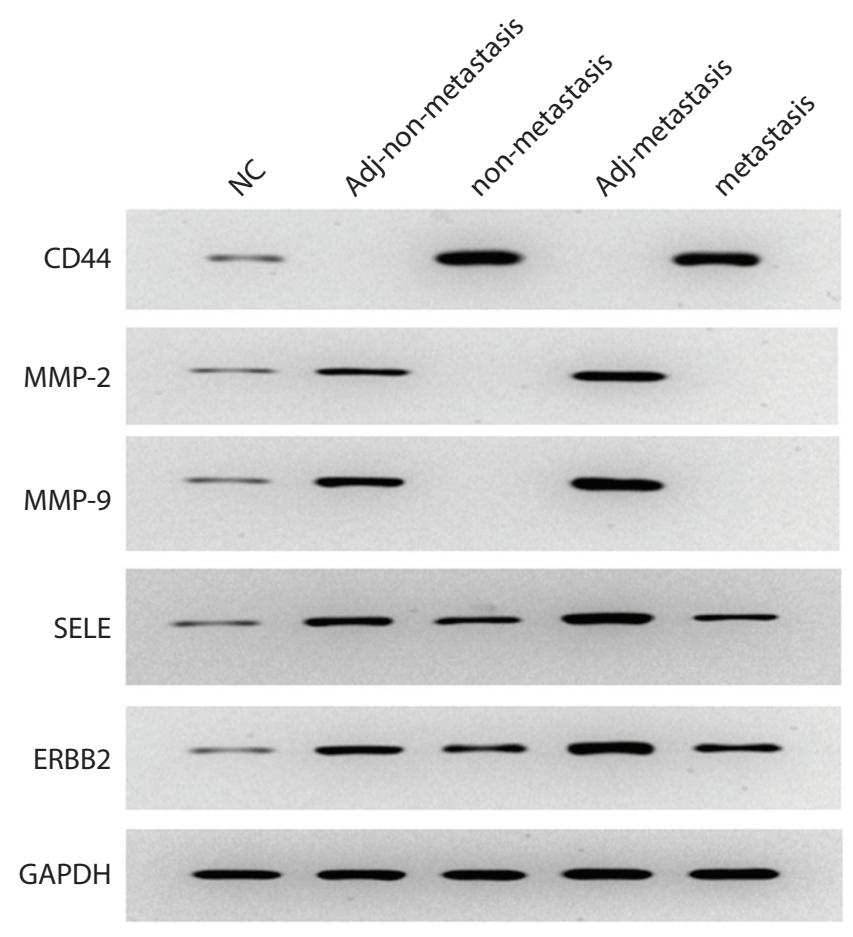

Fig. 4. The protein expression of CD44, HMMR, MMP-2, MMP-9, and SELE in the metastasis, Adj-metastasis, non-metastasis, Adj-non-metastasis, and NC groups was measured with western blotting

of gene therapy, immunotherapy, targeted therapy, and other emerging therapeutic technologies for glioma patients. The mechanisms underlying these therapies need to be elucidated through molecular and pathological experimental 
studies on glioma cells. Therefore, research on the molecular processes of glioma is important. Exosomes have become an area of interest in research over the past few years, and studies have proven that exosomes are a key factor in the occurrence and development of tumors. ${ }^{14}$ Exosomes secreted by tumor cells carry specific small molecules or RNA that can affect the biological functions of other cells and thus regulate the progression of cancer. ${ }^{15}$

Exosomes are cup-shaped vesicles with a double-layer membrane structure, with a diameter of 40 100 nm and a density of $1.13 \sim 1.21 \mathrm{~g} / \mathrm{mL}$. Most of them are located in intercellular spaces or extracellular fluid, or they circulate in the peripheral blood. ${ }^{16}$ Both tumor and non-tumor cells can release a range of cytokines, growth factors, adhesion molecules, and extracellular matrix proteins that mediate cell-cell communication in the tumor microenvironment. ${ }^{17}$ Exosomes are involved in the tumor microenvironment and participate in the signal transduction between cells. ${ }^{18}$ Compared with normal cells, cancer cells secrete larger exosomes, which may be an effective diagnostic marker. ${ }^{19}$ In our study, we isolated and identified exosomes and found that exosomes were abundant in the serum samples of patients with glioma.

The EpCAM is a protein marker of exosomes. To study the mechanism underlying the role of EpCAM in glioma, we determined the expression of CD44, HMMR and MMP-9 with immunohistochemistry in the study groups: metastasis, Adj-metastasis, non-metastasis, Adj-non-metastasis, and NC. The results revealed that CD44 was highly expressed in the non-metastasis and metastasis groups and that the expression of CD44 in the metastasis group was higher than in the non-metastasis group. The levels of HMMR and MMP-9 expression were the highest in the Adj-metastasis and metastasis groups. CD44 is a key receptor of MT1-MMP, which degrades the extracellular matrix. ${ }^{20}$ Expression of CD44 is upregulated in many types of cancer. Moreover, it mediates metastasis by recruiting CD44 to the cell surface. ${ }^{21}$ In colorectal cancer, specific CD44 isoforms are expressed according to the progression of the disease. ${ }^{22} \mathrm{CD} 44$ is a molecular marker associated with cancer stem cell population and treatment resistance in glioma. ${ }^{23}$ The HMMR is found in many cancers, such as colorectal cancer. ${ }^{24}$ It is an oncogene that is highly expressed in glioblastomas and supports the growth of GBM. ${ }^{25}$ Taking this into consideration, we speculated that CD44 was involved in the metastasis of glioma. Our results from western blotting demonstrated significantly higher expression of CD44 in the metastasis and non-metastasis groups in comparison to the $\mathrm{NC}$ group, and higher CD44 expression in the metastasis group than in the nonmetastasis group.

There are some limitations of the current study. Firstly, the sample size was relatively small. Secondly, the regulatory effect of EpCAM in the metastasis of glioma needs to be evaluated in animal studies. The recruitment of CD44 to the surface of glioma cells also needs to be confirmed in vivo.

\section{Conclusions}

We found that the exosome EpCAM promoted the metastasis of glioma by increasing the expression of CD44.

\section{ORCID iDs}

Quan Gu (10 https://orcid.org/0000-0002-5316-4151

Xuelin Chen (1) https://orcid.org/0000-0002-8572-9989

Lin Zhou (1) https://orcid.org/0000-0003-0265-9776

Xianglu Liu (1) https://orcid.org/0000-0001-5276-1467

\section{References}

1. Eckel-Passow JE, Lachance DH, Molinaro AM, et al. Glioma groups based on $1 \mathrm{p} / 19 \mathrm{q}, \mathrm{IDH}$, and TERT promoter mutations in tumors. N Eng J Med. 2015;372(26):2499-2508.

2. Iwadate Y, Matsutani T, Hirono S, Shinozaki N, Saeki N. Transforming growth factor- $\beta$ and stem cell markers are highly expressed around necrotic areas in glioblastoma. J Neurooncol. 2016;129(1):101-107.

3. Louis DN, Perry A, Reifenberger G, et al. The 2016 World Health Organization classification of tumors of the central nervous system: A summary. Acta Neuropathol. 2016;131(6):803-820.

4. Zeng T, Cui D, Gao L. Glioma: An overview of current classifications, characteristics, molecular biology and target therapies. Front Biosci (LandmarkEd). 2015;20:1104-1115.

5. Jeon BN, Yoo JY, Choi WI, Lee $C E$, Yoon HG, Hur MW. Proto-oncogene FBI-1 (Pokemon/ZBTB7A) represses transcription of the tumor suppressor $\mathrm{Rb}$ gene via binding competition with $\mathrm{Sp} 1$ and recruitment of co-repressors. J Biol Chem. 2008;283(48):33199-33210.

6. Kurtz JE, Dufour P. Adecatumumab: An anti-EpCAM monoclonal antibody, from the bench to the bedside. Exp Opin Biol Ther. 2010;10(6): 951-958.

7. Trzpis M, McLaughlin PMJ, de Leij LMFH, Harmsen MC. Epithelial cell adhesion molecule: More than a carcinoma marker and adhesion molecule. Am J Pathol. 2007;171(2):386-395.

8. Went PTH, Lugli A, Meier S, et al. Frequent EpCam protein expression in human carcinomas. Hum Pathol. 2004;35(1):122-128.

9. Maetzel D, Denzel S, Mack B, et al. Nuclear signalling by tumour-associated antigen EpCAM. Nat Cell Biol. 2009;11(2):162-171.

10. Cimino A, Halushka M, Illei P, Wu X, Sukumar S, Argani P. Epithelial cell adhesion molecule (EpCAM) is overexpressed in breast cancer metastases. Breast Cancer Res Treat. 2010;123(3):701-708.

11. Du W, Ji H, Cao S, et al. EpCAM: A potential antimetastatic target for gastric cancer (Retraction of vol 55, pg 2165, 2010). Digest Dis Sci. 2013;58(6):1811.

12. Stoecklein NH, Siegmund A, Scheunemann P, et al. Ep-CAM expression in squamous cell carcinoma of the esophagus: A potential therapeutic target and prognostic marker. BMC Cancer. 2006;6:165.

13. Hu B, Wang Q, Wang YA, et al. Epigenetic activation of WNT5A drives glioblastoma stem cell differentiation and invasive growth. Cell. 2016; 167(5):1281-1295.e18.

14. Kalluri R. The biology and function of exosomes in cancer.J Clin Invest. 2016;126(4):1208-1215.

15. Zhao R, Zhang Y, Zhang $X$, et al. Exosomal long noncoding RNA HOTTIP as potential novel diagnostic and prognostic biomarker test for gastric cancer. Mol Cancer. 2018;17:68.

16. Bang C, Thum T. Exosomes: New players in cell-cell communication. Int J Biochem Cell Biol. 2012;44(11):2060-2064.

17. Sivori S, Falco M, Della Chiesa M, et al. CpG and double-stranded RNA trigger human NK cells by Toll-like receptors: Induction of cytokine release and cytotoxicity against tumors and dendritic cells. Proc NatI Acad SciU S A. 2004;101(27):10116-10121.

18. Ji H, Greening DW, Barnes TW, et al. Proteome profiling of exosomes derived from human primary and metastatic colorectal cancer cells reveal differential expression of key metastatic factors and signal transduction components. Proteomics. 2013;13(10-11):1672-1686.

19. Rabinowits G, Gerçel-Taylor C, Day JM, Taylor DD, Kloecker GH. Exosomal microRNA: A diagnostic marker for lung cancer. Clin Lung Cancer. 2009;10(1):42-46.

20. Kajita M, Itoh Y, Chiba T, et al. Membrane-type 1 matrix metalloproteinase cleaves CD44 and promotes cell migration. J Cell Biol. 2001;153(5):893-904. 
21. Senbanjo LT, Chellaiah MA. CD44: A multifunctional cell surface adhesion receptor is a regulator of progression and metastasis of cancer cells. Front Cell Dev Biol. 2017;5:18.

22. Wielenga VJM, Heider KH, Johan G, et al. Expression of CD44 variant proteins in human colorectal cancer is related to tumor progression. Cancer Res. 1993;53(20):4754-4756.
23. Fu J, Yang Q, Sai K, et al. TGM2 inhibition attenuates ID1 expression in CD44-high glioma-initiating cells. Neuro Oncol. 2013;15(10):1353-1365.

24. Liu W, Ma J, Cheng Y, et al. HMMR antisense RNA 1, a novel long noncoding RNA, regulates the progression of basal-like breast cancer cells. Breast Cancer (Dove Med Press). 2016;8:223-229.

25. $\mathrm{Li} \mathrm{J}$, Ji X, Wang H. Targeting long noncoding RNA HMMR-AS1 suppresses and radiosensitizes glioblastoma. Neoplasia. 2018;20(5):456-466. 